\title{
LE RIFORME ISTITUZIONALI TRA BUONE E CATTIVE
}

\author{
di Giovanni Sartori
}

È difficile che un sistema politico si autoriformi. Ma oramai quasi tutti si sono impegnati nel «fare qualcosa». Tutti o quasi tutti ammettono che il sistema Italia non funziona, o che dovrebbe funzionare meglio. Infine, tutti sottoscrivono un obiettivo comune: uscire dalla democrazia paralizzata aumentando la governabilità, il rendimento e l'efficacia del governare. Se non c'è obiettivo comune non c'è speranza. Ma se c'è, si può sperare. Do per scontato, allora, che esista un fine primario, o prioritario, condiviso un po' da tutti. Le difficoltà restano, e sono di due tipi. Il primo ostacolo è posto dal calcolo dei vantaggi: le parti in causa vogliono riforme che le avvantaggiano (o comunque rifiutano quelle che le penalizzano). Il secondo ostacolo risiede nel calcolo dei mezzi: le parti in causa non sanno calcolare o calcolano male la strumentazione: quali sono i mezzi idonei e sufficienti al fine proposto. Nel primo caso il conflitto è di interessi. Nel secondo caso il problema è di sapere. Discettare di riforme ignorando gli interessi è inutile. Ma è utile mostrare che il calcolo degli interessi, dei vantaggi o svantaggi, può essere sbagliato.

\section{Sistemi elettorali: chiarimenti}

È sempre bene cominciare dal sistema elettorale. È un prima di tutto il resto; e anche se il resto (la riforma costituzionale) non viene, la riforma del sistema elettorale serve anche da sola. Tutti o quasi tutti convengono (in Italia) che il proporzionalismo produce troppa frammentazione partitica, e che questa frammentazione ostacola la governabilità. Ergo, il nostro proporzionalismo deve essere eliminato o quantomeno ridotto. Uno scopo ulteriore della riforma elettorale è, per taluni, di aumentare il potere dei cittadini e di rinsaldare il legame fra rappresentati e rappresen-

RIVISTA ITALIANA DI SCIENZA POLITICA / a. XXI, n. 3, dicembre 1991 
tanti smantellando di pari passo il diaframma dei partiti: è l'idea del «voto pesante» di Pasquino. Lo scopo numero uno - ridurre la frammentazione partitica - è facilmente conseguibile. Lo scopo numero due - attribuire ai cittadini un «voto pesante» - no.

Le proposte di riforma elettorale sono parecchie: una soglia di ammissione-esclusione dalla rappresentanza; il sistema tedesco; l'uninominale; il premio di maggioranza; il doppio turno.

Soglia di esclusione (Sperrklausel). Di per sé è una riforma semplicissima che indubbiamente serve a ridurre la frammentazione decapitando i partiti minori. La difficoltà è di stabilire sensatamente (e di concordare) il livello di esclusione. In Germania è del 5\%; ma in Grecia (che ha giocherellato a oltranza con questo accorgimento) è arrivata ad essere del $25 \%$ per singoli partiti, del $35 \%$ per alleanze a due, e addirittura del $40 \%$ per alleanze di tre o più partiti, attestandosi nel 1985 al 17\%; laddove in Spagna è del $3 \%$. È chiaro che la determinazione della soglia è, in ogni paese, ad hoc. Quale potrebbe essere in Italia? Se del 3\% ucciderebbe soltanto chi dà poca noia (come i liberali); se del $5 \%$ metterebbe a rischio tutti i partiti minori, ma non costituirebbe un ostacolo per le Leghe. La verità è che la clausola di esclusione è un accorgimento riduttivo da introdurre all'inizio. A quaranta e passa anni dalla prima elezione si può imbattere in distribuzioni che la beffano (o che la rendono soltanto iniqua).

Sistema tedesco. È un sistema che viene dichiarato «misto», in parte proporzionale e in parte maggioritario. $\mathrm{Ma}$ il fatto è che per i partiti che superano la soglia del $5 \%$ la proporzione tra voti a seggi risulta, in Germania, perfettamente proporzionale. La efficacia riduttiva (del numero dei partiti) del sistema elettorale tedesco è tutta da attribuire alla Sperrklausel. Se poi ci si chiede come è che una esclusione del $5 \%$ abbia ridotto il numero dei partiti «rilevanti» a tre - democristiani, socialdemocratici e liberali la risposta è che la Sperrklausel da sola non sarebbe riuscita a tanto. Quasi tutti dimenticano che la Corte Costituzionale di Karlsruhe stabilì $a b$ initio che partiti di ideologia comunista e nazista erano anti-democratici e pertanto vietati. Tante grazie; così non è difficile scendere a tre. Ma chi oggi invoca per l'Italia il sistema tedesco mal capisce quel sistema e se ne aspetta benefici che in Italia non produrrebbe perché - clausola di esclusione a parte - il sistema distribuisce i seggi proporzionalmente.

Uninominale. $\grave{E}$ il sistema inglese (mantenuto in Canada, Nuova Zelanda e India) e nordamericano. Uninominale, e cioè «un nome solo», vuol dire che il più votato «piglia tutto». E il più 
delle volte il vincitore che piglia tutto vince con una maggioranza relativa. Se la competizione è, nel singolo collegio, soltanto a due, allora il vincitore vince per forza con la maggioranza assoluta. Ma se $i$ candidati in corsa sono tre o più, allora la maggioranza assoluta non occorre; e tanti più sono $i$ candidati, tanto più la maggioranza che basta per vincere scende. Per esempio, con cinque candidati in corsa si può «prendere tutto» con il $30 \%$ dei voti. Dunque, l'uninominale attribuisce non solo un super-premio di maggioranza, ma lo attribuisce, di regola, a una maggioranza relativa: il che produce, al minimo, un super-premio del $51 \%$. Chi grida alla truffa contro il premio di maggioranza proposto dalla Dc dovrebbe mettere senz'altro in carcere chi propone l'uninominale.

Il premio di maggioranza. Qui l'intento è solo di correggere il proporzionalismo. Pertanto il premio in questione si limita a sovrarappresentare e, viceversa, a sottorappresentare; ma, in principio, non decapita nessuno. Occorre peraltro distinguere tra premio di maggioranza attribuito a una maggioranza assoluta, e premio attribuito alla maggioranza relativa. Nel primo caso si rafforza una maggioranza che è già tale; nel secondo si trasforma in maggioranza la «minoranza maggiore». Ma neanche in questo caso è corretto parlare di truffa. Un premio attribuito alla maggioranza relativa ripete il caso più frequente dell'uninominale con questa importante attenuazione: che pur sempre consente la sopravvivenza dei partiti minori che l'uninominale stritola. E nemmeno è vero che il premio di maggioranza penalizza i partiti minori. Se incentiva, come dovrebbe, apparentamenti elettorali, allora premia anche (in proporzione) $\mathrm{i}$ partiti minori della coalizione vincente.

Doppio turno. Non si sa perché, in Italia è invalso l'uso di chiamare il doppio turno «sistema maggioritario a doppio turno»; dizione che è ad un tempo sbagliata e fuorviante. Infatti il doppio turno non deve essere, al ballottaggio, uninominale; può essere, anche, plurinominale.

Mettiamo che venga adottato un doppio turno trinominale (che elegge i primi tre). In tal caso il doppio turno funziona come un sistema proporzionale (che vota persone, non liste) frenato e corretto dalla piccolezza del collegio. $\mathrm{Ma}$, allora, perché non mantenere la proporzionale operando soltanto sulla dimensione delle circoscrizioni? Risposta: perché nell'ipotesi di collegi piccolissimi (trinominali o quadrinominali, come in Giappone) votare due volte a ragion veduta è molto più razionale e meno distorcente che non votare alla cieca una volta sola. Nell'ipotesi consi- 
derata non c'è nemmeno ragione di escludere nessuno dall'accesso al secondo turno: essendo la distribuzione dei voti già nota, $i$ candidati senza speranza sparirebbero per debolezza propria e ai loro elettori (nel primo turno) viene offerta la opportunità di votare per una seconda preferenza, o comunque per il male minore. Così la frammentazione partitica viene ridotta senza che nessuno abbia diritto di lamentarsi.

$\mathrm{Ma}$ se niente vieta che il doppio turno sia plurinominale, niente egualmente vieta che sia uninominale. Anche così la differenza fra uninominale «secca» (a un colpo solo) e uninominale in seconda votazione resta grandissima. Intanto il doppio turno può imporre, volendo, la maggioranza assoluta che l'uninominale non può imporre: basta che al ballottaggio siano ammessi soltanto $\mathrm{i}$ due candidati più votati al primo turno. $\mathrm{Ma}$ il doppio turno è anche atto a produrre un vincitore a maggioranza relativa che sia «meno minoritario» di quello prodotto - a parità di candidati in corsa - dall'uninominale secca. In tal caso si tratterà di graduare il livello della soglia di ammissione (al secondo turno). In Francia si esordì con il $6 \%$, per poi arrivare al $12,5 \%$. Ma quale che sia la soglia, il vantaggio sempre è che un elettore che vota due volte riorienta il proprio voto. Lo ridico: il votare diventa più razionale e il risentimento dell'elettore che perde viene attutito.

\section{Sistemi elettorali: valutazioni}

Entrando nel dibattito, i casi da dibattere si possono ridurre a tre: uninominale, premio di maggioranza, e doppio turno. Io sono contrario, per l'Italia, all'uninominale; sconsiglio, ma soprattutto per motivi di circostanza, il premio di maggioranza; e raccomando il doppio turno.

Critica dell'uninominale. Cominciamo dai pregi. Dico subito che se l'India tiene ed è riuscita sinora a funzionare, è proprio in virtù dell'uninominale. Se l'India adottasse la proporzionale, sprofonderebbe rapidamente in una polverizzazione senza rimedio. Del pari è l'uninominale che blocca, a livello di sistema politico, le tensioni etniche e razziali che sempre più traversano la società americana da quando il melting pot, il calderone di fusione che ha creato in passato la «nazione» americana, ha cessato di fondere alcunché. Infine, convengo che se si punta su un sistema bipartitico di tipo inglese, occorre sostenerlo con l'uninominale. (Ma non convengo, come si vedrà, che basti l'uninominale a pro- 
durlo). Dunque, non sono per nulla ostile all'uninominale in linea di principio. Ma l'unico merito certo dell'uninominale è di essere il sistema elettorale più «forte» e cioè più riduttivo e manipolativo di tutti ${ }^{1}$. Gli altri meriti attribuiti all'uninominale sono invece incerti e indebitamente gonfiati.

Gli argomenti dei fautori dell'uninominale sono essenzialmente tre: i) che rafforza il legame «diretto» tra elettori ed eletti, ii) che l'elettorato insedia direttamente, con il suo voto, il governo, iii) che migliora, visto che si votano persone e non liste di partito, la qualità del personale parlamentare. Li possiamo ritrascrivere, alla Pasquino, così: che l'uninominale crea un voto «pesante», che pesa più del voto proporzionale, $i$ ) in peso rappresentativo, ii) in peso governativo, iii) in peso qualitativo. Questi vantaggi dovrebbero compensare la controindicazione (sulla quale gli uninominalisti sono inclini a sorvolare) che l'uninominale riduce la scelta «efficace» dell'elettore a una sola alternativa; il che da tempo irrita molto gli inglesi, che hanno dato il $25 \%$ dei loro voti ai liberali (nel 1983) per trovarsi beffati con un magrissimo $3.5 \%$ dei seggi (il «peso» del voto liberale è stato così dieci volte minore del peso del voto laburista). Ma vediamo partitamente.

i) Il legame «diretto» tra elettori ed eletti è, tanto per cominciare, un legame ridotto a zero per quel $40-60 \%$ dei votanti che «perdono tutto». $\grave{E}$ anche dubbio, in secondo luogo, che il rappresentante si senta tenuto a servire l'intera maggioranza (relativa o assoluta) che lo elegge. Di solito, l'elezione è assicurata da piccoli elettorati strategici che controllano lo scarto - mettiamo un 5-10 per cento - che separa il vincente dai perdenti. Pertanto l'uninominale finisce per privilegiare - per servire più e meglio - $\mathrm{i}$ sotto-elettorati identificabili (e mobilitabili in blocco) che assicurano la vittoria. Sarebbe, in Italia, un paradiso per le zone mafiose (altro che controllo mafioso del voto di preferenza!).

Ciò premesso, la variabile interveniente decisiva è se il partito è debole (come in USA), o forte (come in Inghilterra). Se è debole, all'americana, allora l'uninominale produce, o tende a produrre, un gretto «localismo circoscrizionale» (nemmeno, si badi, regionale) che frantuma un paese in mille egoismi ${ }^{2}$. Se il partito è forte, all'inglese, allora il «rapporto diretto» lascia il tempo che trova: il backbencher è ferreamente diretto dal partito (non dai

\footnotetext{
${ }^{1}$ Vedi per esteso sub «Sistemi Elettorali» in Sartori (1990, 245-258 e passim) dove preciso anche la nozione di sistema elettorale «forte» e «debole».

2 Il punto è sviluppato sub «Videopotere» in Sartori (1990), spec. pp. 302-306.
} 
suoi elettori) ed è tenuto a sostenere compattamente il proprio governo. Dunque, il rafforzamento del legame «diretto» tra elettori ed eletti è - quando avviene - di dubbia desiderabilità: gli inconvenienti sono grossi. Inoltre, se si dà - in ipotesi - un voto pesante che rafforza la rappresentanza (rendendola più diretta), in tal caso non si dà un voto pesante, atto a meglio sostenere il governo. Le due istanze si escludono l'una con l'altra.

ii) Passiamo all'argomento che il sistema uninominale elegge anche il governo che governerà per tutta la legislatura. Duverger parla al riguardo di «democrazia immediata»; ma è una etichetta sbagliata che fa sbagliare. In realtà quella immediatezza è mediata, mediatissima. Duverger e coloro che lo seguono dimenticano che l'uninominale opera collegio per collegio, che la sua efficacia manipolativa è circoscrizionale. Pertanto se ci sono cento collegi e in ciascuno vince un partito diverso (il candidato di un partito che vince solo lì, solo una volta), ne risulterà un parlamento di cento partiti. Dunque, l'uninominale produce un sistema nazionale di tipo bipartitico solo se gli stessi due partiti sono in condizione di vincere in tutti i collegi. Il che vuol dire che il fattore decisivo è la strutturazione a due del sistema partitico a livello nazionale. $\mathrm{Ne}$ consegue che se manca questa condizione, questo fattore intermedio, non è più vero che l'uninominale insedia direttamente il governo. Difatti, non sarebbe vero in Italia.

Lo spiego immaginando uno scenario. Se in Italia venisse adottato il sistema uninominale, non è improbabile che la prima volta (un po' per imperizia, e parecchio per dispetto) i candidati che si presenterebbero in ciascun collegio siano quattro o cinque. In tal caso la Dc con il $34 \%$ dei voti nazionali potrebbe vincere da sola anche il $90 \%$ dei seggi. Se accadesse, la lezione sarebbe davvero memorabile per gli sconfitti; tanto memorabile da indurli, la volta dopo, a coalizzarsi tutti quanti all'insegna di un fronte laico. Perché no? Caduto il comunismo, cade anche - lungo la dimensione destra-sinistra - la polarizzazione che poneva il confine ad excludendum: l'ex Pci diventa perfettamente includibile in una alleanza che va dai liberali al Pds includendo socialisti, socialdemocratici e repubblicani. In tal caso la Dc si troverebbe fronteggiata, in ogni collegio, da un solo candidato. Poniamo che questo fronte laico arrivi a mettere assieme il $40 \%$ dei voti nazionali. Se così, potrebbe vincere il $90 \%$ dei seggi, e sarebbe la Dc, questa volta, a prendere il tonfo. $\mathrm{Ma}$, attenzione, il governo della seconda legislatura generata dall'uninominale non sarebbe monocolore ma di coalizione: una coalizione tutta da discutere (a cinque, a quattro, 
a tre?), che può cadere anche ogni anno, e che può anche variare. L'Italia non sarebbe come l'Inghilterra, ma semmai come l'India. Beninteso, non succederà. Tanto più lo scenario sopra immaginato apparirà credibile, tanto più si tradurrà in una profezia che si autodistrugge. Resta che la cosiddetta democrazia «immediata» prodotta dall'uninominale non è per nulla un effetto diretto e automatico del sistema elettorale.

iii) L'ultimo argomento è che la personalizzazione uninominale del voto migliora la qualità degli eletti. Sì e no. Ai fini del miglioramento dei candidati il miglior sistema è di gran lunga il doppio turno (che richiede trasferimenti di elettorati da un partito ad un altro, quindi violazioni di «identificazioni partitiche», e quindi trasferimenti che i partiti non possono più comandare). Con l'uninominale (secca) la personalità-qualità del candidato diventa importante solo nei collegi «insicuri» nei quali lo scarto tra vincitore e sconfitto è di pochi punti percentuali; ma non lo è nei collegi sicuri.

Tirando le somme, e tornando al «voto pesante» di Pasquino, confesso che non ci credo. In parte non ci credo perché, se e quando si dà (come negli Stati Uniti), è controproducente. $\mathrm{Ma}$ non ci credo per la ragione di fondo che il cosiddetto voto pesante imputa concretezza a aggregati astratti. Il discorso concreto è che il peso del voto di ciascuno è espresso da una frazione il cui denominatore è il quoziente che dà elezione. Se il quoziente è di cinquantamila voti, il mio voto pesa un cinquantamillesimo. Voto pesante? No: voto piuma. Nel mondo reale nel quale viviamo il voto pesante è travolto dai grandi numeri. Pertanto discutere $\mathrm{i}$ pro e i contro dei sistemi elettorali nell'ottica suggerita da $\mathrm{Pa}$ squino è, secondo me, discuterli male.

Il premio di maggioranza: si ma no. Ho già detto che dichiarare il premio di maggioranza «truffaldino» è polemica poco seria. $\mathrm{Ma}$ è serio rifiutarlo con l'argomento che in Italia andrebbe a congelare una situazione che è invece, oggi, in movimento. La sinistra italiana si è fluidificata, si sta riallineando e anche, quantomeno in grossa parte, avvicinando al centro. Come già notavo, la nostra dimensione destra-sinistra si è accorciata e si sta normalizzando: la sua polarizzazione non è più - nella classe del pluripartitismo estremo - anomala. Pertanto un gioco «a largo campo» (nella sua differenza da quello, avventuristico, a tutto campo) è il gioco che meglio consente la ristrutturazione del sistema. In particolare il Psi ha ragione, mi sembra, di non volere un sistema elettorale che lo incastra nei confronti dell'elettorato di sinistra che 
deve pur cercare di catturare. Per entrambe queste ragioni non vedo perché si debba insistere sulla formula del premio di maggioranza quando esiste - passo ora ad argomentare - una alternativa migliore ed accettabile per tutti: il doppio turno.

Il doppio turno: si. Vediamo prima il doppio turno maggioritario (uninominale). In tal caso si può stabilire che al ballottaggio siano ammessi soltanto i due «più votati» del primo turno. Questo è il sistema usato in Francia per l'elezione presidenziale, ed è senz'altro il sistema da raccomandare a quel fine. Produce un presidente eletto a maggioranza assoluta, e alla lunga ingenera un allineamento bipolare (e, in Francia, depolarizzato) dello schieramento partitico. $\mathrm{Ma}$ il meccanismo può essere reso più soffice $\mathrm{e}$ più flessibile in sede di elezione di corpi collegiali (parlamenti, consigli regionali e comunali, e simili). In tal caso tra il primo e il secondo turno basta interporre un «filtro». Vuoi che si ricorra, a questo fine, a una soglia di esclusione (il 6\% iniziale dei Francesi potrebbe anche essere un filtro funzionale per l'Italia), oppure che si stabilisca un numero fisso di ammessi al ballottaggio (in Italia, per esempio, i primi cinque), in ogni caso è importante lasciar passare tanti candidati quanti bastano a un duplice fine: $i$ ) consentire che al primo turno l'elettorato sia davvero «libero» di esprimere la sua prima preferenza e, ii) rendere necessario che al ballottaggio i partiti «trattino» ritiri concordati in compensazione.

L'importanza del primo punto è intuitiva, e chiarisce il grandissimo vantaggio del doppio turno uninominale sull'uninominale secca: se l'elettore è «coercito» a votare, nel ballottaggio, per una sua seconda/terza preferenza o anche, in dannatissima ipotesi, per il male minore, questa coercizione non è da imputare al sistema elettorale ma invece alla volontà «in maggioranza» degli altri elettori. Una bella differenza. L'importanza del secondo punto è, invece, che modera la campagna elettorale e fabbrica un «collante» atto a produrre maggiore coesione o comunque minore litigiosità nei governi di coalizione che si andranno a formare. Siccome i partiti sanno che a distanza di una o due settimane dal primo voto si dovranno «passare» tra loro $\mathrm{i}$ rispettivi elettorati (e così candidati che implicitamente dicono: «mi ritiro in favore di»), ne risulta che si dovranno aggregare in famiglie di cugini piuttosto che in accozzaglie di diavoli.

Notavo che il premio di maggioranza è controindicato in situazioni fluide, «in movimento». Il doppio turno non si espone alla stessa obiezione; anzi facilita le osmosi contrastando, al tempo stesso, la polverizzazione incoraggiata, nei momenti di di- 
sgelo, dalla proporzionale. Dicevo anche, all'inizio, che il massimo ostacolo nel quale si imbattono in pratica le riforme è che ogni partito ne precalcola perdite e profitti (per sé). Il doppio turno non consente precalcolazioni: è protetto dal «velo di ignoranza» di Rawls. Nella «posizione originaria» ipotizzata da Rawls nessuno è in grado di prevedere in quale situazione si andrà a trovare nelle posizioni successive. Pertanto adotterà una strategia maximin: assicurarsi il massimo dei vantaggi minimi e, viceversa, minimizzare al massimo il possibile danno. Insomma, è razionale, per chi deve decidere più o meno alla cieca, minimizzare il rischio. E siccome il doppio turno avvantaggerà chi saprà giocare meglio il nuovo gioco, è il sistema elettorale che più soddisfa la razionalità rawlsiana: siccome nessuno è certo ex ante di perdere o vincere, è la scelta meno rischiosa.

Quanto al doppio turno plurinominale, anche in questo caso il vantaggio è che l'elettore è coercito, nel non poter votare per la sua prima preferenza, solo dalla distribuzione dei voti che scopre al primo turno. Il secondo vantaggio, quello di produrre un «collante», è meno sicuro. Ma esiste qui un terzo vantaggio: la decapitazione uninominale viene attutita da una proporzionalizzazione che pur sempre riduce la frammentazione partitica. E il doppio turno plurinominale è protetto più che mai dal velo dell'ignoranza.

\section{Elezione diretta: stabilità, effettività e legittimità}

I sistemi elettorali sono modificabili, in Italia, per legge ordinaria. Inoltre, non sono riforme costituzionali nel senso che non investono la struttura dello Stato. Ma tra riforme costituzionali e no si interpone una zona che appartiene ad entrambi i contesti: la elezione diretta che insedia ad una carica esecutiva. Pertanto la demo-investitura costituisce un elemento isolabile dell'insieme delle riforme istituzionali. Il tema è da enucleare anche per l'ulteriore ragione che traversa l'alternativa tra presidenzialismo e parlamentarismo. Se l'elezione diretta è del capo dello Stato, allora il sistema diventa di tipo presidenziale. Se l'elezione diretta è del capo del governo, allora il sistema resta di tipo parlamentare. $\mathrm{Ma}$ l'elezione diretta è invocata - quale che sia l'assetto sistemico anche per i sindaci, e in generale al livello delle amministrazioni locali.

L'idea è in ogni caso di rafforzare il potere di decidere, e dunque la tesi è che la demo-investitura, la elezione diretta, aiuta la 
governabilità. Vero? Quel che è vero è che l'elezione popolare diretta produce stabilità: per tutta la durata del mandato l'eletto resta in carica, non cambia. Ma la stabilità produce effettività? No, la risposta è nettamente no. La stabilità non è condizione sufficiente di effettività. Se lo scopo dell'elezione diretta è di ottenere un esecutivo efficiente e capace di decidere, allora l'elezione diretta di un sindaco, oppure del primo ministro, oppure del capo dello Stato, non basta e non è nemmeno detto che aiuti. Durare è un conto, potestas decidendi è tutt'altro conto. Ed è chiaro che in Italia i proponenti dell'elezione diretta non sono in chiaro su questa differenza.

Esiste anche una controindicazione generale alla elezione diretta: siccome dà stabilità, non consente di rimediare l'errore. Se l'eletto (ad una carica) è un imbecille, un inetto, un cialtrone, o quant'altro, ce lo dobbiamo egualmente godere per 4-5 anni. I demo-elezionisti non hanno mai risposto a questa obiezione, e di regola la ignorano. Ma il loro far finta di non vedere ne conferma la forza.

I due punti di cui sopra valgono, in generale, per qualsiasi livello di elezione diretta (locale, governativa o presidenziale). $\mathrm{Ma}$ pesano diversamente ai vari livelli. Inoltre, man mano che il livello cresce entra in gioco un ulteriore elemento: che la demo-elezione «in carica» attribuisce un più di legittimità. Per un sindaco questa «aggiunta di legittimità» non aggiunge granché; ma aggiunge molto ad un primo ministro insediato a volere di popolo; ed è determinante per la creazione di un sistema presidenziale.

A livello di amministrazione locale, e soprattutto per i piccoli comuni nei quali i cittadini (gli abitanti delle città) si conoscono faccia a faccia, la elezione diretta del sindaco è probabilmente una buona idea. Nelle grandi città pro e contro si bilanciano. Negli Stati Uniti i sindaci sono eletti direttamente; ma mi sfugge perché milanesi o romani si troverebbero meglio amministrati se importassero l'attuale sindaco di New York (un buon uomo di smisurata inettitudine), oppure quello di Washington (un ribaldo cacciato soltanto perché condannato per droga). I contro aumentano a dismisura, invece, se si contempla l'elezione diretta del presidente del Consiglio: una idea che a me sembra del tutto peregrina.

L'uomo sbagliato reso inamovibile può essere digerito a livello locale, ma è una sciagura innecessaria e pertanto stupidissima a livello nazionale. Il plus di legittimità non gli serve a nulla (salvo che a garantirne la durata), visto che i poteri del presidente del Consiglio sono in ogni caso i poteri che gli attribuisce la costitu- 
zione, e che gli vengono «materialmente» dalla costellazione delle forze che lo appoggiano o avversano. Siccome non è nemmeno detto che il capo del governo demo-eletto esprima una maggioranza o sia il più adatto a procurarsela, egli rischia di essere impallinato in tutte le sue decisioni ancor più e ancor meglio di un presidente del Consiglio di creazione parlamentare. Nei sistemi partitici all'italiana, il solo «sblocco» dei governi bloccati è cambiarli; il che vuol dire che l'efficienza (relativa) dei nostri governi è semmai prodotta dalla loro instabilità, non dalla loro stabilità. Insomma, l'idea del presidente del Consiglio demo-eletto si imbatte soltanto in controindicazioni. Non solo rende irrimediabile la scelta sbagliata, ma confonde stabilità con efficienza. Un capo del governo demo-eletto che regna senza poter governare, cosa fa? $\mathrm{Si}$ appella al paese? Gli serve a poco, perché un presidente del Consiglio non può agire, come un presidente della Repubblica, da solo: l'organo è collegiale, non individuale. Alcune riforme non sono da fare perché inutili. Questa non è da fare perché dannosa.

Si dirà che le controindicazioni che valgono per la elezione diretta del capo del governo, valgono anche per la elezione diretta del capo dello Stato. Ma sarebbe una inferenza precipitosa. Dipenderà da quale sistema presidenziale andremo a costruire.

\section{Sistemi presidenziali}

Un sistema presidenziale non può essere tale, per definizione, se il capo dello Stato non è creato da una elezione popolare diretta (o assimilabile a una elezione diretta) ${ }^{3}$. In prima approssimazione, allora, i pregi e difetti del presidenzialismo dovrebbero essere i pregi e difetti di qualsiasi demo-insediamento. Controlliamo se è vero. Primo: l'elezione popolare diretta dà stabilità, ma non conferisce di per sé alcun potere. Vero? Sì. Nel caso dei presidenti dell'Austria, Irlanda, Islanda, la loro elezione popolare diretta non toglie che restino presidenti largamente cerimoniali. $\grave{E}$ così - si osserverà - perché la costituzione li lascia senza poteri. $\mathrm{Ma}$ i presidenti latino-americani sono invece presidenti costituzionalmente potenti, eppure il risultato non cambia di molto:

\footnotetext{
${ }^{3}$ E il caso degli Stati Uniti o dei paesi nei quali il parlamento si limita a sanzionare la scelta popolare nel caso dei presidenti eletti a maggioranza relativa (come per Allende in Cile). L'assimilabilità diventa dubbia, invece, nel caso della Bolivia, dove il parlamento esercita la facoltà di scegliere tra i primi tre eletti.
} 
sono spesso presidenti fortissimi sulla carta, ma pur sempre deboli, di regola, in termini di effettività ${ }^{4}$.

Secondo: l'elezione diretta di un presidente aggrava, rendendola immodificabile, la scelta sbagliata. Vero? Ovviamente sì. Il punto non è se l'elezione popolare produca scelte migliori o peggiori delle elezioni demandate ai corpi rappresentativi, ai parlamenti. Ammettiamo che nei due casi le probabilità di errore, o di cattiva scelta, siano uguali. La differenza resta che nei sistemi non-presidenziali governa un governo cambiabile, mentre nei sistemi presidenziali governa un presidente non cambiabile (fino alla scadenza del suo mandato). Nel caso del presidente sbagliato l'errore diventa così irrimediabile (o rimediabile soltanto a colpi di golpe ${ }^{5}$. Il punto viene ampliato, nelle critiche di Linz e Valenzuela, così: i sistemi presidenziali sono caratterizzati da «rigidità» mentre i sistemi parlamentari sono caratterizzati da flessibilità ${ }^{6}$. Sì; ma la rigidità che rende difettosi i presidenzialismi fa capo a questo difetto di impianto: la inamovibilità della persona sbagliata.

Terzo: l'elezione popolare diretta conferisce una legittimità «personale» e quindi un extra di legittimità. Vero quando questa è l'intenzione (e dunque non sempre). Ma la legittimità da sola non risolve (e può anche essere una mina vagante). Gioverà o meno a seconda dei contesti nei quali si inserisce.

Il presidenzialismo puro. Fin qui abbiamo considerato soltanto il denominatore comune di tutti i possibili sistemi presidenziali: l'elezione popolare del capo dello Stato. Liberiamoci ora dei casi irrilevanti: Austria, Irlanda e Islanda. Li ho richiamati per sottolineare che dalla sola elezione popolare di un presidente (il suggerimento iniziale di Craxi) non segue e consegue nulla. $\mathrm{Ma}$ di quei casi ci possiamo sbarazzare riclassificandoli come sistemi parlamentari anomali nei quali il capo dello Stato non è eletto dal parlamento. Veniamo così alla analisi di insieme dei presidenziali-

\footnotetext{
4 In generale (e con eccezioni) il presidente latino-americano nomina a tutte le cariche non-elettive e controlla le spoglie; ma questo potere «di palazzo» si ferma alle soglie del parlamento, dove il suo potere è soltanto di gestire la «piccola politica» quotidiana. Se tenta la «grande politica» delle riforme e delle innovazioni, allora viene bloccato, e il presidenzialismo latino-americano oscilla tra l'impotenza e il deragliamento.

${ }^{5} \mathrm{Di}$ regola il golpe è militare e instaura regimi militari. I suoi effetti sono ancora più nocivi, peraltro, quando è il presidente che si trasforma in dittatore: è segnatamente il caso di Peron in Argentina e Vargas in Brasile. L'eredità di Peron ha disastrato l'Argentina per mezzo secolo e il Brasile è uscito del tutto frantumato - in chiave di governabilità - dalla sequenza Vargas-Goulart-regime militare (1964-1985). Il Presidente in carica, Collor, è un presidente televisivo che galleggia su un Congresso di partiti volatili.

6. Cfr. Juan Linz (1991); e Arturo Valenzuela (1991).
} 
smi genuini cominciando, come si deve, dal prototipo: il sistema presidenziale di tipo americano, e cioè il caso del presidenzialismo «puro».

Il sistema americano si fonda sul principio della separazione dei poteri: il presidente governa, il parlamento controlla. È una soluzione che ha la forza (grandissima) della sua semplicità. Ma questa semplicità si complica man mano che si instaura la prassi del «governare legiferando». Se gli atti di governo devono assumere la forma di legge, allora il presidente non può governare senza il concorso dei legislatori. Il governo è sì diviso dal parlamento (non è di emanazione parlamentare); ma il governare diventa, nel fatto, condiviso. Se non è condiviso, e cioè se il presidente non ha una sua maggioranza in parlamento, allora il governare si inceppa: il governo non cade, ma cadono i suoi atti. Dunque il presidenzialismo americano postula, per funzionare, la maggioranza indivisa: che una stessa maggioranza vinca la presidenza e anche il controllo del parlamento. Negli Stati Uniti è stato così quasi senza eccezione fino al 1954. Da allora, e sinora, quel trend si è rovesciato: per due terzi del tempo i presidenti si sono imbattuti, in parlamento, in una maggioranza contraria. E così l'America del Nord comincia ad avere i problemi che hanno paralizzato la sua progenie: il presidenzialismo dell'America del Sud. Si capisce che gli Stati Uniti fruiscono pur sempre della «rendita» di due secoli di avviamento, di successo e di mestiere. È un vantaggio inestimabile. Fruiscono anche del vantaggio di tutta una serie di circostanze facilitanti, a cominciare dalla flessibilità pragmatica della politica americana. Ma si tratta di una costellazione di circostanze largamente irripetibili.

La prima condizione di «funzionalità» del presidenzialismo puro è, si è già detto, la maggioranza indivisa. Come si ottiene? Si ottiene - seconda condizione - da un sistema bipartitico, cioè da un allineamento delle forze partitiche che si dispieghi in termini di pura e semplice maggioranza e minoranza. Il presidente è «in maggioranza» in quanto esiste un partito - il suo - che ottiene la maggioranza assoluta dei seggi. Si badi: una maggioranza «di coalizione» non servirebbe, o comunque servirebbe male. Così come la carica presidenziale è indivisibile, anche la maggioranza presidenziale in parlamento deve essere indivisa (monopartitica). Non basta. A questo punto occorre guardare al partito: deve essere indisciplinato oppure dovrebbe essere disciplinato?

A rigore di logica la terza condizione dovrebbe essere che occorre il partito disciplinato (all'inglese). Sì: è la situazione otti- 
male nell'ipotesi della maggioranza indivisa (il presidente in maggioranza al parlamento). Allora sì che il presidenzialismo può essere forte. Senonché il partito disciplinato diventa condizione disastrante nell'ipotesi della maggioranza divisa (il presidente in minoranza). In tal caso il partito disciplinato creerebbe una perfetta paralisi di potere. Pertanto la indisciplina dei partiti americani fornisce la valvola di sicurezza del sistema: consente al presidente minoritario di «comprarsi» (si fa per dire) di volta in volta una maggioranza. Talvolta gli riesce, talvolta no; ma è così che il sistema riesce ancora a funzionare, seppur precariamente, con presidenti minoritari. Il succo è questo: che la indisciplina dei partiti parlamentari degli Stati Uniti frena le «presidenze forti» (il presidente in maggioranza è pur sempre meno forte di quanto non si creda) e salva le «presidenze deboli» (i presidenti di minoranza). -Dovrei allora concludere che la terza condizione di funzionalità del presidenzialismo puro è un bipartitismo indisciplinato? Esito.

Esito perché se passiamo ai presidenzialismi dell'America Latina a formato più o meno bipartitico ${ }^{7}$, diventa chiaro che la loro debolezza deriva in non piccola parte dal frazionismo o fazionalismo interno dei partiti, e quindi della loro indisciplina. D'altra parte, è ugualmente chiaro che nel caso di maggioranza divisa, e ancor più nei casi di frammentazione partitica, l'indisciplina è utile. Il presidenzialismo puro è così difficile da gestire da richiedere una navigazione a vista: non possiamo prestabilire se il partito che più gli conviene debba essere forte o debole, coesivo o sconnesso. La terza condizione è incerta.

Comunque, due condizioni sono certe: maggioranza indivisa e strutturazione bipartitica. Se queste condizioni non sono soddisfatte, allora il sistema presidenziale entra in difficoltà: funziona a un basso tasso di effettività o non funziona per nulla. Dei venti paesi circa dell'America Latina, nell'ultimo mezzo secolo nemmeno uno si è salvato da almeno un golpe militare (il più «continuo», il Costa Rica, dura dal 1949). Non sarà soltanto colpa del

\footnotetext{
${ }^{7}$ Dico più o meno bipartitico perché in almeno due casi - Uruguay e Colombia - $\mathrm{i}$ veri attori sono $\mathrm{i}$ sotto-partiti (lemas in Uruguay, e dai jefes naturales alle roscas in Colombia). La Colombia batte tutti in anomalia: due partiti «ufficiali» che si combattono, alle elezioni, presentando sino a otto liste distinte! I casi credibili di bipartitismo si riducono pertanto al Venezuela e all'Argentina. Il Venezuela ha sperimentato due volte presidenti minoritari (nel 1968 e 1978), con partiti che sono oggi da caratterizzare come disciplinati. L'Argentina ha invece ottenuto (dal ricominciamento del 1983) una maggioranza indivisa: Alfonsin con maggioranza radicale e Menem con maggioranza peronista. Entrambi sono, o sono stati, presidenti che non possono contare sui rispettivi partiti più di tanto.
} 
presidenzialismo: ma venti su venti è troppo, non può essere una correlazione spuria. Anche se i regimi dell'America Latina sono «precari» per molte e diverse ragioni (storiche, culturali, sociali, economiche e altre), una di queste è sicuramente l'esistenza di strutture politiche che sono mal strutturate.

Il semi-presidenzialismo. La domanda subito è: in che modo il semi-presidenzialismo modifica il presidenzialismo puro eliminandone o correggendone i difetti? La caratteristica che va a definire tutti i semi-presidenzialismi è che non si fondano sulla separazione dei poteri ma invece ricorrono a un potere intermedio: un governo che si interpone tra presidente e parlamento e che attende al «governare legiferando», e cioè al raccordo tra iniziativa presidenziale e approvazione parlamentare.

Quando si dice semi-presidenzialismo si pensa alla V Repubblica, al sistema francese inaugurato da De Gaulle. Ma anche la Repubblica di Weimar (1919-1933) fu a suo modo un sistema semi-presidenziale. E per meglio capire il semi-presidenzialismo conviene tenere a mente anche la formula tedesca ${ }^{x}$. Vediamo allora di definire il semi-presidenzialismo in generale. La prima caratteristica resta che il capo dello Stato è di elezione popolare (diretta o «come se» diretta). La seconda caratteristica è invece inedita: il governo non è più il governo «personale» del presidente, ma un organo costituzionale a sé che per taluni rispetti assomiglia ai governi dei sistemi parlamentari. Vuoi che il capo dello Stato presieda i lavori del governo o no, in ogni caso la carica di capo del governo è una carica pleno iure. Pertanto il semi-presidenzialismo è tale in quanto prevede un esecutivo diarchico. Una complicazione? No; a guardare bene una complicazione non è.

$\mathrm{Si}$ capisce che anche al semi-presidenzialismo si pone il problema della maggioranza divisa; e si capisce che anche il semi-presidenzialismo funziona al meglio nel caso della maggioranza indivisa. $\mathrm{Ma}$, intanto, il semi-presidenzialismo si può fabbricare una maggioranza in un modo non consentito al presidenzialismo puro. In virtù dello sdoppiamento dell'esecutivo, non occorre più che la maggioranza che sostiene il governo presidenziale sia dello stesso partito o monopartitica. Il presidente è sempre uno (la carica è indivisibile), ma nulla vieta che il suo governo sia plurimo, e cioè un governo di coalizione che associa al potere tanti partiti

\footnotetext{
${ }^{8}$ Debbo rinviare, sul punto, al «Corriere della Sera» del 19 luglio 1991, dove riva. luto, tra l'altro, la costituzione di Weimar.
} 
quanti occorrono per «fare maggioranza» (in parlamento). Dunque, un presidente che non consegue una sua maggioranza parlamentare elettoralmente, può ancora rimediare fabbricandosi una maggioranza parlamentare governativamente. Ceteris paribus, qui la situazione avversa della maggioranza divisa è sanabile.

$\mathrm{Ma}$ vediamo più a fondo. Anche nel caso ottimale della maggioranza indivisa lo sdoppiamento dell'esecutivo non può nuocere, dal momento che la diarchia in questione è gerarchizzata: il presidente è il motore primario che nomina e revoca a sua discrezione il capo del governo e largamente condiziona, nel fatto se non anche in diritto, la scelta dei ministri. D'altra parte lo sdoppiamento dell'esecutivo giova in ogni caso a rendere più efficace l'esercizio del potere presidenziale. L'esistenza di un governo che operi come cinghia di trasmissione della volontà presidenziale attribuisce al presidente francese una incidenza sui lavori parlamentari che fa vistosamente difetto al presidente americano.

Veniamo alla ipotesi della maggioranza divisa. In tal caso la struttura diarchica perde in parte (ma non del tutto) la sua direzione gerarchica e si trasforma, in parte, in coabitazione. $\mathrm{Ma} \mathrm{Du}$ verger è precipitoso quando equipara questa situazione a una trasformazione del presidenzialismo in parlamentarismo. Intanto Duverger dimentica la legittimità autonoma che caratterizza un presidente demo-eletto e che manca al presidente di elezione parlamentare. Dimentica anche tutte le prerogative che Mitterrand ha mantenuto, visto che gli spettano costituzionalmente, nel periodo di coabitazione con Chirac. No. Il bello del semi-presidenzialismo è che si adatta alla circostanza avversa della maggioranza divisa; e adattarsi non è trasformarsi in altro.

Il punto è che mentre il sistema puro è - tra presidente e parlamento - «senza ponte», il semi-presidenzialismo si impernia, appunto, su un ponte che è ad un tempo di transito e di snodo. Il punto è, dunque, che il semi-presidenzialismo è dotato di una flessibilità che il modello americano non possiede. Ma sostenere che il semi-presidenzialismo sia migliore del presidenzialismo puro non è sostenere che la formula francese sia di sicuro funzionamento. Questa sicurezza non c'è. Difatti andrò a proporre un semi-presidenzialismo nel quale il «semi» sta per un presidenzialismo «alternante»: una formula - sosterrò - atta a risolvere $\mathrm{i}$ problemi che le altre formule (presidenziali e no) non risolvono. Ma di questo in appendice. Prima devo proseguire l'esame dei regimi che esistono. 


\section{Sistemi parlamentari}

I sistemi parlamentari sono tali in quanto si fondano sul principio che il parlamento è sovrano. Ne consegue che i governi sono insediati e abbattuti dal parlamento, e che anche il capo dello Stato è di regola (ma non inderogabilmente; e può anche essere un monarca) di elezione parlamentare. Nei casi nei quali il parlamentarismo ammette un presidente demo-eletto, non gli conferisce poteri né gli riconosce una legittimità distintiva: quel presidente resta «parlamentare» perché resta sottoposto al principio della sovranità del parlamento.

Peraltro dalla sovranità del parlamento possono poi discendere in concreto sistemi molto diversi che suddivido - per simmetria di insieme e in prima approssimazione - tra sistemi di parlamentarismo puro e sistemi semi-parlamentari. Il parlamentarismo puro è tale nella misura nella quale si risolve in un sistema di onnipotenza assembleare, e quindi di appiattimento e di confusione dei poteri; mentre il semi-parlamentarismo è tale nel senso che dimezza il potere del parlamento per ristabilire un soprastante e distinto potere di governo. Ma nel mondo reale il parlamentarismo puro (assembleare) finisce per essere ancor meno vitale e ancora più raro del presidenzialismo puro. Per sopravvivere diventa lestamente impuro, e cioè corretto e in qualche modo ridotto a ragione. Il che non vuol dire che si trasforma in semi-parlamentarismo. I casi finiscono dunque per essere tre: parlamentarismo puro, impuro e dimezzato. Per semplicità e maggiore chiarezza dirò assembleare il parlamentarismo puro (estremo), e dirò parlamentarismo (senza specificare) per indicare il tipo impuro o normale.

Parlamentarismo e partitocrazia. Il caso del parlamentarismo assembleare (puro) è esemplificato per antonomasia dalla III Repubblica Francese - la ben detta «repubblica dei deputati» - e può essere raffigurato come l'esatto rovescio del presidenzialismo puro: un meccanismo monomotore per il quale, questa volta, è il parlamento che dovrebbe muovere tutto. A questo modo il difetto si inverte: così come il presidente «solo» non passa nel governare, analogamente il parlamento «solo» eccelle nel malgovernare e nel produrre ingovernabilità.

La differenza è che mentre il presidenzialismo è rigido, il parlamentarismo è flessibile, e così trova modo di funzionare generando i propri anticorpi: insomma, trasformandosi in un parlamentarismo non-assembleare. Come? Di solito lasciandosi imbri- 
gliare (di fatto, ché si tratta di una prassi non disegnata da nessuno) dal controllo e dalla disciplina del partito. Il parlamentarismo viene reso agibile, dunque, dalla partitocrazia, dal potere parlamentare (sui parlamentari) dei partiti. Le partitocrazie, vedremo, sono parecchie. Alcune sono difendibili, altre no. Ma anche a voler considerare la «partitocrazia parlamentare» un male, è pur sempre un male necessario: blocca l'assemblearismo. Anche così il parlamentarismo all'italiana dura vivacchiando e senza forza di ricupero. Così chi rifiuta, in Italia, i rimedi di tipo presidenziale punta oggi, in alternativa, sul semi-parlamentarismo di tipo tedesco o inglese.

Il semi-parlamentarismo. I sistemi semi-parlamentari modificano il parlamentarismo in analogia a come il semi-presidenzialismo modifica il presidenzialismo: sono anch'essi sistemi a due motori nei quali il governo tiene testa (Germania) o anche prevale (Inghilterra e simili) sul parlamento. Dico semi-parlamentarismo per dire, dunque, che l'onnipotenza del parlamento viene grosso modo dimezzata. Dal che non consegue, si badi, che il semi-parlamentarismo produca un parlamento impotente: ché se lo depotenzia nel potere di intralciare il governo, lo potenzia nella funzione di controllo. Si guardi all'Inghilterra. Lì il parlamento ricupera - in sede di opposizione - quel potere di controllo che nei sistemi parlamentari si dissolve, nel fatto, in un cogobierno collusivo: il parlamento «complice» che copre la colonizzazione e lottizzazione dell'economia, il finanziamento occulto dei partiti e, insomma, tutto il vero «malaffare di Stato» (del quale è partecipe). In Inghilterra non sarebbe possibile: l'opposizione davvero vigila, e il question time ai Comuni è un efficacissimo strumento di vigilanza.

Ma procediamo con ordine. Il caso inglese è da esaminare per primo perché è il caso forte della categoria. Nei testi che ripetono stancamente le dizioni della metà dell' 800 , l'Inghilterra figura sotto la voce «governo parlamentare». Ma no; il sistema reale è invece, da tempo, un sistema di premiership e di governo di gabinetto (e dunque di governo non-parlamentare) nel quale il motore prevalente è il «motore governo». Ai fini dell'effettività il sistema inglese è davvero il sistema ideale. Ma come ci si arriva? Quali sono le condizioni che lo consentono?

Intanto, e per cominciare, ci vuole il partito che accetta il principio della leadership e che ne viene strutturato in modo conforme. In secondo luogo occorre un governo monopartitico risultante da un sistema bipartitico posto da due soli partiti «rilevanti»» 
ciascuno dei quali può e vuole governare da solo (in alternanza con l'altro) ${ }^{9}$. I casi importanti di sistemi bipartitici (non-presidenziali) sono l'Inghilterra, la Nuova Zelanda e, in approssimazione, il Canadà. Siccome nessuno dei tre reggerebbe un giorno se il proprio bipartitismo non fosse sostenuto dall'uninominale, ne risulta che l'uninominale è quasi sempre un presupposto sine qua non. $\mathrm{Ma}$ non ne consegue che l'uninominale sia condizione sufficiente di bipartitismo. L'India è uninominale e lontanissima dall'essere bipartitica; se l'Italia adottasse l'uninominale (si è già visto) non diventerebbe per questo bipartitica; e nemmeno lo è del tutto il Canadà, dove l'uninominale spesso non dà la maggioranza assoluta dei seggi a nessuno e insedia governi monocolori di minoranza. E il Canadà spiega bene la natura del sistema.

Infatti, come mai il Canadà preferisce un monocolore minoritario a coalizioni (a due) che metterebbero il governo in maggioranza? La risposta è che per il sistema in questione il governo monopartitico è condizione più necessaria dell'altra. Infatti è il governare da solo (nell'alternativa: o solo io, o solo te) che produce eo ipso la coesione (oltre che la ferrea disciplina) del partito parlamentare che sostiene il suo governo. In Inghilterra (e casi simili) rovesciare il proprio governo non si traduce nel rifarne un altro; significa regalare il governo al partito di opposizione. L'operazione sarebbe tutta e soltanto in perdita. E questo è il «segreto» che fa funzionare il sistema inglese.

Passiamo alla'Kanzlerdemokratie tedesca. È un caso attenuato di semi-parlamentarismo, nel senso che non si fonda sulla preminenza, ma piuttosto sulla autonomia del governo nei confronti del parlamento. A questa attenuazione corrisponde peraltro una maggiore elasticità: il sistema consente coalizioni a due. Ma dubito che reggerebbe con governi di coalizione più estesi. Perché? Di nuovo la risposta è nelle condizioni che consentono la cosiddetta democrazia del cancelliere.

S'intende che anche il sistema del cancellierato si fonda prima condizione - sulla accettazione del principio di leadership. $\mathrm{Ma}$ in Inghilterra la leadership nasce dall'interno del partito, mentre in Germania è la carica che si riflette sul partito. La differenza è sfumata, ma fa differenza. Il cancelliere tedesco non è, come il premier inglese, un «primo sopra ineguali»; ma è pur sempre un

\footnotetext{
9 Per una definizione più precisa e l'analisi del formato e della meccanica bipartitica, vedi Sartori (1982, 74-78).
} 
«primo tra ineguali» che può rimpastare il governo senza cadere, e che cade soltanto a séguito del voto di sfiducia costruttivo. Il punto resta che prima occorre, come condizione fondante, la preminenza del cancelliere-leader. Senza questo prius il rinforzo del voto di sfiducia costruttivo non rinforza nulla: da solo, quell'argine è di carta (e in Italia aggirarlo sarebbe un gioco da ragazzi).

La seconda condizione è, nel caso tedesco, un sistema tripartitico. Meglio ancora, è ovvio, se il sistema fosse bipartitico; ma dubito che il cancellierato tedesco potrebbe reggere se richiedesse coalizioni a tre, specie se $i$ tre partners fossero di forze relativamente eguali (mettiamo 25, 20 e 15 per cento). La regola di massima mi sembra questa: che nei governi di coalizione la forza del cancelliere capo del governo finisce largamente ai confini del proprio partito. Se il partito minore è piccolo - mettiamo tra il 5$10 \%$ - e se il secondo partito non si può permettere di essere ballerino (i liberali tedeschi hanno cambiato alleanza, ad oggi, una volta sola e dichiarano prima di ogni elezione la loro intenzione di alleanza), allora è chiaro che il cancelliere in carica non può essere disobbedito e ricattato più di tanto. Ma se i soci del partito del cancelliere diventassero due, e se il gioco a tutto campo - con diverse possibili combinazioni di alleanza - diventasse possibile, allora cancellierato addio.

La condizione diventa, allora, la riduzione dei partiti «rilevanti» a tre. Si ritiene che questa riduzione sia stata conseguita, in Germania, dal sistema elettorale. $\mathrm{Ma}$ - già lo si è visto - a torto. La riduzione a tre dei partiti tedeschi non deriva per nulla dalla mal capita natura «mista» del sistema elettorale, ma invece dalla clausola di esclusione (che quasi fece sparire i liberali nel 1969) più l'esclusione dei comunisti e nazisti in forza dell'articolo 21 della Legge Fondamentale.

La verità è, temo, che il semi-parlamentarismo è difficile da fabbricare: è, tra tutte, la formula che più sfugge alla presa della ingegneria costituzionale. Al tempo stesso il semi-parlamentarismo è caratteristicamente fragile. Nel caso inglese la sua forza è un po' come la forza del presidenzialismo americano: è forza di lunga pratica e di «convenzioni» della costituzione. Nel caso tedesco la fragilità è manifesta: per mettere il sistema del cancellierato in crisi basta un riallineamento elettorale che renda determinanti o comunque «rilevanti» $\mathrm{i}$ Verdi o un nuovo partito che emerga dalla ex Germania dell'Est.

I sistemi a partito predominante.: Duverger, dopo aver inventato la «democrazia immediata», ci vende ora le «democrazie 
compiute». Quali sarebbero? Nel testo dal quale cito («Corriere della Sera» del 29 luglio 1991) sarebbero Francia, Inghilterra, Germania, Svezia, Norvegia, Austria, Spagna. Che cosa le renda compiute (si badi, come democrazie) a me resta oscuro. Ma compiute o no, è chiaro che Duverger ha in mente le democrazie «che decidono», le democrazie efficienti. Tra queste Duverger curiosamente dimentica il Giappone, che aggiungo io. Allora, a che titolo sono efficienti? Duverger richiama il sistema elettorale e l'alternanza. Ma cominciamo dal mettere il suo guazzabuglio in ordine.

La Francia è semi-presidenziale e Inghilterra e Germania sono semi-parlamentari. La loro efficienza è da spiegare così. Ma gli altri paesi sono parlamentari. Cosa è che li rende democrazie che decidono? Il sistema elettorale? Non può essere. L'Irlanda si avvale del singolo voto trasferibile; il Giappone fa caso a sé (piccoli collegi che eleggono, in media, i primi quattro candidati più votati); Norvegia e Svezia sono paesi proporzionalisti a oltranza; Spagna e Austria, invece, paesi a proporzionalismo frenato. Dunque, $\mathrm{i}$ paesi in questione coprono quasi tutto lo spettro dei sistemi elettorali. E coprono anche, noto per inciso, un largo spettro di frammentazione partitica: dai due-tre partiti dell'Irlanda, Austria e Giappone, ai quattro-cinque di Norvegia e Svezia. Quanto all'alternanza, a questo effetto Duverger sbaglia ancora di più: tutti i suoi paesi parlamentari (Irlanda, Giappone, Norvegia, Svezia, Spagna, Austria) sono stati «efficienti» in quanto e quando caratterizzati da poca o nessuna alternanza, e cioè quando hanno funzionato come sistemi «predominanti» di governo monopartitico $^{10}$. E la loro efficienza è da ascrivere, più che ad ogni altro singolo fattore, alla caratteristica di avere, o avere avuto, monocolori sorretti da una maggioranza assoluta (per parecchie legislature) in parlamento.

Qui non mi interessa discutere se sia vero che nel caso italiano (che differisce da quelli succitati perché la Dc non è un partito predominante, e quindi è costretta a governare in coalizione) il gioco dei fattori si inverte: e cioè se sia vero che la non alternanza è il male, e l'alternanza il toccasana. Il semplicissimo punto da fermare è che la governabilità può semplicemente risultare dal sistema partitico, dal suo strutturarsi come un sistema a partito predominante. $\mathrm{Ma}$ il nostro sistema partitico non è di questo tipo. $\mathrm{E}$

\footnotetext{
${ }^{10}$ Sui sistemi a partito predominante (da non confondere con l'esistenza di partiti dominanti come la Dc italiana), vedi di nuovo Sartori $(1982,68-70)$.
} 
se la governabilità non dipende necessariamente o soltanto dalla forma dello Stato, sicuramente non dipende dai fattori indicati da Duverger.

Il parlamentarismo migliorato. Anche senza riformare il sistema, si possono sempre fare cose utili per migliorare il sistema. Abolire il voto segreto fu utile. Sarà utile che il broglio delle preferenze sia impedito dalla necessità di scrivere il nome del preferito sulla scheda. Il lavoro legislativo può essere snellito e enormemente alleggerito - come propone Spadolini - dall'adozione del principio del «silenzio assenso» (di una camera rispetto alle deliberazioni dell'altra). Del pari - come propone Maccanico si deve precisare e limitare la «riserva legislativa» del parlamento; il che vuol dire che occorre delegificare una indigesta e immensa Babele di leggi da restituire all'ambito regolamentare.

Ancora: tutte le proposte attese a ostacolare la «corsa alla spesa» che avviene, specie sotto elezioni, nelle aule parlamentari sono tutte proposte benemerite. Che il parlamento non possa modificare i provvedimenti finanziari predisposti dal governo (come da tempo viene chiesto da Andreatta); che al capo dello Stato sia attribuito il potere-dovere di veto «linea per linea» (line item veto) della spesa di dubbia copertura; che la Banca d'Italia acquisti la stessa autonomia della Bundesbank tedesca o della Federal Reserve americana; che alla Corte dei Conti sia consentito, dopo aver inutilmente denunziato al vento, di adire, quando ne ricorrono gli estremi, al potere giudiziario; questi rimedi e simili sono tutti da perseguire, riforma costituzionale o no. Infine, i regolamenti delle Camere, specie di Montecitorio, sono ancora largamente da smantellare e rifare. Io li ritengo importantissimi.

E dunque non sottovaluto affatto l'importanza delle cosiddette piccole riforme, delle riforme correttive e migliorative. Ho sostenuto da sempre che fare qualcosa è meglio di non fare nulla, e che le «grandi riforme» sono per molti il grande pretesto per bloccare tutto. Tutto ciò concesso non sarà il parlamentarismo migliorato a «spaccare la macchina» che occorre spaccare.

\section{La maccbina}

Le macchine sono automatismi e la politica italiana si costituisce in macchina appunto perché è diventata un meccanismo perverso che gira, sciupa e divora per moto proprio, in caduta inerziale. Fa poca differenza, oramai, volere o non volere la macchina. 
Anche chi non la vuole ne viene inghiottito; e chi ne esce, esce di scena.

Giuliano Amato non la chiama macchina, ma la sua denunzia del «governo spartitorio» ne individua un elemento centrale. Un altro elemento centrale è peraltro la «colonizzazione», il dilagare aberrante dei partiti extra moenia. Che quel dilagare alla conquista del potere economico e delle sue risorse sia spartitorio e lottizzante non toglie che sia - a titolo proprio - occupazione di uno spazio, di uno spazio improprio. C'è poi un fattore moltiplicante: lottizzazione e colonizzazione avvengono a due livelli, prima a livello di partito, e poi di sottopartito (di frazioni e fazioni). Così la spartizione tra partiti è moltiplicata e aggravata dall'appetito interno di partito. Finisce che per ogni spoglia gli aventi diritto (nel diritto ufficioso dei partiti) sono diecine. E per soddisfare chi resta a terra bisogna colonizzare e conquistare altre spoglie, e così senza fine e confine. E un ciclo infernale di «spettanze». A volte la macchina sosta perché anche ai più impudenti viene la paura di strafare, perché c'è crisi, perché i carrozzoni di Stato perdono davvero troppo. Ma poi la paura passa e la macchina si rimette in moto.

Tutti deprecano la macchina. Ma anche chi la depreca sul serio accetta l'argomento che l'occupazione partitica dello spazio economico sia una necessità di tutte le democrazie. Ma non è vero. Sì, la politica «costa», e quindi tutte le democrazie devono trovare un modo di finanziarla. Ma la politica costa infinitamente meno in Inghilterra che in Italia (e quindi c'è modo di bloccarne e ridurne il costo). Inoltre, la politica può essere finanziata in parecchi modi, e il modo italiano è non solo anomalo ma il peggiore di tutti. In Giappone la politica non costa meno che in Italia, ma i politici giapponesi non hanno colonizzato il proprio sistema economico. Gli americani ancor meno, e cioè per nulla. E gli americani riescono, in più, a imporre la trasparenza dei finanziamenti politici. Non dico che le soluzioni di altri paesi non abbiano i loro inconvenienti; ma hanno l'inestimabile pregio di non strangolare il processo economico. E dunque è falso che la colonizzazione partitica dell'economia sia un male inevitabile che affligge più $o$ meno tutte le democrazie. No: la «via italiana» di finanziare la politica è non solo tra le più dannose, ma anche una via che la maggioranza delle democrazie serie non ha imboccato.

$\mathrm{Ma}$ perché dico «macchina» e non dico, come dicono i più, partitocrazia? La riposta è implicita in quanto sopra, ma vale esplicitarla. Il potere dei partiti è molteplice, si manifesta in sedi 
diverse, e a diverso titolo. Ho già menzionato, per esempio, la partitocrazia parlamentare e «disciplinante» che emenda il parlamentarismo assembleare. Piaccia o no ( $\mathrm{mi}$ irriterebbe molto, ne sono sicuro, se io fossi un peone a Montecitorio o un backbencher a Westminster), a me sembra necessaria. Difendo anche, a fronte dello spappolamento dei partiti americani, la necessità del partito «forte» (anche al centro). Dunque, non dobbiamo fare di ogni erba un fascio. Se le partitocrazie le vogliamo ammazzare tutte, a questo modo le lasciamo tutte in vita: i bambini proteggono l'acqua sporca. Il bersaglio deve essere individuato. E l'acqua sporca - il bersaglio da centrare - è soprattutto la colonizzazione spartitoria, e cioè la partitocrazia parassitaria che munge la vacca dello Stato, poi del parastato, e infine tutte le vacche che riesce ad arraffare.

Come ci liberiamo, allora, della macchina? Amato spera che il semi-presidenzialismo di tipo francese ci liberi quantomeno del «governo spartitorio». Al che Leopoldo Elia obietta, mi sembra a ragione, che «l'esperienza francese non ci rassicura sull'acquisizione del principale vantaggio che Amato ci prospetta come effetto del semi-presidenzialismo e cioè il superamento e l'eliminazione dei gravissimi danni provocati dalla partitocrazia» ${ }^{11}$. Sì; ma poi la Dc propone, per combattere i «gravissimi danni provocati dalla partitocrazia» (e confido che Elia concorderebbe con la mia precisazione su quali siano), rimedi ancora più blandi, e sicuramente ancora meno efficaci, di quelli proposti dai socialisti. Alla fine viene il dubbio che nessuna delle riforme esaminate sinora alla macchina arrivi mai.

Si avverta: non è che la macchina sia oramai indistruttibile perché è diventata, per il nostro politico, condizione di vita. Così gli può sembrare; ma è altrettanto vero che ci sarebbe vita, e vita migliore, senza la macchina. La macchina risucchia e ingoia i propri utenti. Liberarsi della macchina e del suo vortice sarebbe un sollievo. Se la macchina non c'è più, e se non c'è per nessuno, tutti restano pari. Pari e più distesi. Se per sopravvivere è obbligatorio rubare, tutti devono rubare. Ma se rubare diventa impossibile $\mathrm{i}$ più (i non-ladri di natura) dormirebbero meglio. Se una singola elezione non costa più tre miliardi ma trecento milioni (tariffa di tipo inglese), viene meno l'affanno di dover ar-

${ }^{11}$ Cfr. «Il Politico», 1991, II, pp. 207 ss. 
raffare tre miliardi. È questione di riabituarsi. Riabituarsi al peggio è difficile. Riabituarsi al meglio no.

\section{Appendice: Il presidenzialismo alternante}

Ricapitoliamo, ripartendo dalla specularità (invertita) tra presidenzialismo e parlamentarismo. Entrambi sono sistemi monomotori, ma nel primo il motore è collocato in alto, al vertice dello Stato, e viene fiaccato dall'attraversamento discendente del parlamento, mentre nel secondo il motore sta in basso, è il parlamento, e sfilaccia, in salita, il governare. Assai più spesso che no, il presidenzialismo è un governare che non passa, e il parlamentarismo un governare che non decolla. I due sistemi funzionano solo in virtù di circostanze aiutanti: per il presidenzialismo la maggioranza indivisa; per il parlamentarismo il partito predominante. Circostanze che non possono essere assicurate dalla ingegneria costituzionale.

I sistemi misti sono tali perché a due motori. Per chi parte, come gli italiani, dal parlamentarismo, viene facile preferire il semi-parlamentarismo, che viene percepito come una correzione migliorativa del sistema che ci è noto. $\mathrm{Ma}$, si è visto, i sistemi semi-parlamentari di tipo inglese o tedesco sono ancor meno costituzionalizzabili dei sistemi monomotori: farli nascere e funzionare per fiat costituzionale è pressoché impossibile. Lo abbiamo capito per il sistema inglese più che per il cancellierato tedesco. Pertanto insisto: $\mathrm{i}$ conti che facciamo in Italia sulla riproducibilità della Kanzlerdemocratie sono, a conti fatti, conti mal fatti. Resta allora il semi-presidenzialismo. E la grande forza di questa soluzione è di essere, tra tutti i sistemi considerati, il più costituzionalizzabile. Nemmeno qui l'ingegneria costituzionale può tutto; ma può fare ottanta invece di quaranta. Ma anche il semi-presidenzialismo si può incagliare nelle secche della maggioranza divisa. Inoltre non romperebbe «la macchina»: a questo fine la cura è troppo blanda. Possibile che non si possa fare meglio? Mi provo a sostenere di sì.

I sistemi semi-presidenziali sono sistemi a due motori simulta$n e i$; ma perciò nulla garantisce che i due motori siano sintonizzati e si aiutino l'uno con l'altro. Il sistema che propongo di alternanza tra parlamentarismo e presidenzialismo è sì, anch'esso, un congegno a due motori; ma di due motori in successione. Siccome 
la mia proposta è già stata largamente esposta ${ }^{12}$, mi limito a ricordare in breve che prevede che ogni legislatura esordisca come un normale sistema parlamentare (quello che di fatto abbiamo) al quale vengono consentiti due governi; dopodiché, caduto il secondo, viene attivato ope legis, in sostituzione compensativa e punitiva, il motore presidenziale. Ma non potrà il limite dei «due governi» venire aggirato da un gioco di rimpasti? È la prima obiezione, alla quale subito rispondo che per impedire questo escamotage basta che il capo dello Stato non firmi le nomine dei ministri «rimpastati». Dunque l'idea non è più di miscelare due sistemi in uno (come nel semi-presidenzialismo), ma invece di utilizzarli alternativamente come correttivi reciproci. S'intende che l'alternanza basta che sia «minacciata»; e tanto più risulterà punitiva, tanto più i punibili cercheranno di renderla innecessaria.

Il meccanismo sopra descritto non è - a dispetto di come può apparire a primissima vista - un meccanismo complicato. $\mathrm{Al}$ contrario. È semplice perché non è condizionato da nessuna condizione. Prende quel che trova con i difetti che ha. Se il parlamentarismo, sotto minaccia, si emenda, l'obiettivo della governabilità è conseguito. Se no, l'impotenza del parlamentarismo viene compensata pro tempore da un presidenzialismo potenziato. Siccome ogni sistema porta inevitabilmente con sé i difetti dei propri pregi, il ritrovato è di rimediare i difetti dell'uno - in rotazione - mediante i contro-difetti dell'altro.

Un salto nel buio? Così dicono molti critici; ma, ritengo, a torto. Parlamentarismo e presidenzialismo sono sistemi notissimi e capiti da chi li pratica. Il guaio è che chi conosce bene l'uno in genere mal conosce e mal capisce l'altro. Ma accoppiare due cose note non produce buio. Il buio è prodotto dall'ignoranza, e cioè affligge chi conosce un solo sistema. $\grave{\mathrm{E}}$, dunque, un buio rimediabile.

Ma ammettiamo che la mia sia una «improvvisazione» insufficientemente meditata. Può darsi. Vediamo; cominciando dal perché siamo a corto di idee. Una ovvia risposta è che le idee (nuove idee) non possono venire da un formalismo che attende soltanto alla «logica giuridica» delle sue costruzioni. A questo modo si resta, inevitabilmente, all'interno dell'ordinamento che abbiamo.

${ }^{12}$ La prima formulazione è apparsa sul «Corriere della Sera» del 26 aprile 1991. Agli interventi che ha provocato ho risposto sul «Corriere della Sera» del 4 maggio, del $1^{\circ}$ giugno, e del 19 luglio. «Il Politico» (II, 1991) ha successivamente pubblicato un mio testo introduttivo e il dibattito di Amato, Elia e Barbera avvenuto alla sala Zuccari del Senato, estendendolo a Galeotti, Manzella e Miglio. 
D'altra parte il costituzionalismo che ha creato, nel corso del '700 e dell' 800 , le costituzioni garantistiche si preoccupava, prima di tutto e soprattutto, di limitare e controllare il potere. Non ho mai preso questa preoccupazione sottogamba ${ }^{13}$, e la considero pur sempre primaria. Ma la costituzione formale «vive» e viene man mano modificata dalla costituzione materiale. E la costituzione materiale ci è sempre più scappata di mano: è diventata la resa del giurista al fatto compiuto. Il quesito diventa allora come mai non sappiamo controllare, e tantomeno pilotare, l'andamento (pur denunziato come degenerativo e perverso) della costituzione materiale. Quesito al quale rispondo che ci siamo dimenticati del sistema di incentivi. Il mondo reale non è regolato soltanto da divieti e comandi. Tra quel che le norme giuridiche vietano o prescrivono (comandano di fare o prescrivono come sia da fare) si interpone un vastissimo territorio «libero», e cioè regolato da incentivazioni, da meccanismi informali di ricompensa e, viceversa, di privazione.

Un po', dunque, ho meditato, arrivando a questa conclusione: che sempre più il giurista ha guardato all'ufficio dimenticando le persone (in ufficio). Dal che ricavo che quantomeno de iure condendo, quantomeno in sede costituente, il giurista che ignora le motivazioni e i meccanismi di incentivazione è destinato a bucare l'acqua. Pertanto, e per contro, la mia proposta è tutta incentrata sulle aspettative di «persone reali» che si chiedono: cosa mi avvantaggia e, viceversa, cosa mi danneggia? Beninteso, nell'ambito delle strutture che le vincolano e nelle quali sono obbligate a muoversi.

E tempo che entri nel vivo. Comincio dal ribadire che il mio progetto di presidenzialismo alternante presuppone che il regime normale, di base, sia un regime parlamentare. Augusto Barbera è molto turbato dal sospetto - insiste nel dirmelo - che la mia sia una proposta «in salsa cilena» ${ }^{14}$. Se lo fosse, non vedo che cosa ci sarebbe di male. Ma non lo può essere. Infatti io prevedo che lo scatto che insedia il sistema presidenziale sia attivato dalla seconda caduta di un governo di investitura parlamentare. Barbera rifletta: se partiamo (come in Cile) da un sistema presidenziale, quale può essere l'automatismo che lo fa decadere? Proprio non saprei. Inoltre mi sfugge come un parlamentarismo sostitutivo del

13 Vedi a conferma sub «Costituzione» in Sartori (1990, 11-24).

14 Cf. «L'Unità» del 27 marzo 1991, e poi «Corriere della Sera» del 13 giugno 1991. 
presidenzialismo ne andrebbe a correggere i difetti. Se il presidenzialismo decade perché impotente, il parlamentarismo (probabilmente assembleare) che andrebbe a sostituirlo sarebbe ancora più impotente. Barbera si rassicuri una volta per tutte: la salsa che raccomando in America Latina è la salsa semi-presidenziale $^{15}$. $\mathrm{E}$ in Cile ho detto: se volete (ma ve lo sconsiglio) un sistema parlamentare, allora prevedetelo come un sistema «punibile». Ma non ho detto: se avete uno zoppo, allora rimediate con un altro zoppo. Quando mi accorgo di una sciocchezza, non la dico.

Un secondo ordine di precisazioni riguarda la fase presidenziale della formula del presidenzialismo alternante. Siccome l'alternanza è una eventualità, il mio disegno potrebbe essere detto «presidenzialismo di riserva». Ma siccome il mio motore di riserva deve essere un motorone, dichiararlo «di riserva» sarebbe una blandizia e un infingimento (alla Duverger). No. Il mio dovrà essere, quando richiamato in servizio effettivo, un presidente «plenipotenziario» (nei limiti che dirò). Se no, no. Un presidente bloccabile non risolverebbe niente. È solo un presidente messo in grado di operare contro un parlamento ostruzionistico che rende inutile quell'ostruzionismo e semmai utile (per il parlamento) di collaborare.

Una precisazione concomitante è che un sistema di presidenzialismo alternativo richiede tassativamente un presidente demoeletto in coincidenza con la durata della legislatura. Se la legislatura è di cinque anni, la presidenza è di cinque anni: parlamento e presidente sono eletti simultaneamente e decadono simultaneamente. Va da sé che nel mio meccanismo non c'è ragione di contemplare lo scioglimento anticipato del parlamento (questo è un potere che il presidente non avrebbe). Per contro la rielezione del presidente deve essere consentita: il sistema di incentivi largamente dipende da questa condizione. Infine, siccome a un presidente plenipotenziario occorre una legittimità non contestabile, deve risultare eletto dalla maggioranza assoluta.

Mettiamo ora in moto lo scenario (ambientato, si intende, in Italia). Il secondo governo parlamentare, poniamo nel terzo anno della legislatura, è ormai logoro; e siccome si profilano le elezioni, le tensioni pre-elettorali si aggravano. Inutile andare avanti, o stare fermi, così. Il governo viene lasciato cadere, forse anche con

15 Difatti questa è la tesi di fondo del mio scritto «Né Presidenzialismo né Parlamentarismo» (Sartori 1991), che traduce un testo inglese concepito e destinato a un volume sul Sudamerica. 
sollievo (le patate bollenti passano ad altri), scatta il sistema presidenziale, e il presidente nomina lui, davvero a sua scelta, un governo che il parlamento non può rovesciare. Nemmeno lo può negoziare, perché è tassativamente prevista l'incompatibilità: i ministri del governo presidenziale non possono essere parlamentari. Defunge così sia il manuale Cencelli come la caccia alle poltrone. Quanto alle posizioni di potere del parastato, quelle che mano mano vengono a scadere potranno essere rinnovate da un presidente che si giocherebbe la rielezione se non attendesse a disfare, nei limiti, il malfatto. Alla fine del suo mandato, su cento cavalli di Caligola che ha trovato all'IRI e dintorni ne resteranno, mettiamo, settanta. Un taglio alla volta, dopo un ciclo di presidenze «la macchina» rientrerebbe in limiti di abuso accettabili.

$\mathrm{Si}$ intende che il «ben fare» del presidente risulta (dovrebbe risultare) dal sistema di incentivi e dalle aspettative che ne accompagnano l'avvento. Un presidente che diventa governante dopo essere stato più o meno a lungo sotto ghiaccio, si trova d'un tratto sotto gli occhi di tutti in un contesto precisato e pressante di aspettative: ci si aspetta da lui che «faccia», che sblocchi le situazioni bloccate, che tagli i nodi che il parlamentarismo non sa affrontare, e soprattutto che esca dal solco del malfare che sono tutti costretti, volenti o nolenti, a praticare: il malfare imposto dalla «macchina». Non si può avvalere del pretesto di «non potere»: può. E non ha alibi, non si può nascondere dietro la cortina fumogena dei governi di coalizione: lui e soltanto lui è responsabile. Infine, se ha affrontato e vinto la maratona di una elezione popolare, si deve presumere che sia motivato, e che sia motivato anche a «riuscire». Un riuscire premiato - è importantissimo dalla rieleggibilità.

Quali le controindicazioni? La più grave sarebbe, se credibile, che il mio sistema è «pericoloso» perché rischia di allevare un presidente-dittatore. Ho già risposto a diverse riprese a questa obiezione ${ }^{16}$. Se la durata della legislatura è di cinque anni, è ragionevole prevedere che in media il presidente sarà plenipotenziario per circa due anni. Scaduta la legislatura rientra automaticamente nei ranghi, il suo «scettro» si dissolve come la carrozza di Cenerentola. Per quanto forte, sarà sempre forte a breve. Quando scade, cosa potrebbe fare? Inforcare il cavallo bianco, chiamare a

16 Vedi «Il Corriere della Sera» del 4 maggio 1991, nel quale mi richiamo a un ragionevole calcolo del rischio, e poi del 19 giugno 1991 . 
raccolta i forestali, i gladisti, e i servizi segreti e così impadronirsi dello Stato? Abbiamo visto, di recente, regimi ritenuti inespugnabili fondersi come la neve al sole non perché non avessero armi, ma perché diventati insicuri della propria legittimità. Peccare di ottimismo è per me esperienza inedita. Ma per una volta peccherò. L'eventualità, oggi, in Italia, di un dittatore illegittimo che conquisti il potere con la forza, e che si regga sulla forza delle baionette (quali?), è una eventualità che non mi toglie nemmeno un minuto di sonno.

Una diversa controindicazione - di Angelo Panebianco e poi di altri - è che il marchingegno venga manipolato da un «partito del presidente», e cioè da una coalizione trasversale di forze interessate a fare scattare al più presto possibile il presidenzialismo. $\mathrm{Ma}$ quanto può essere, arguendo, il più presto possibile? Dopo due anni? Anche se così fosse, un presidente triennale rientra, direi, nelle tolleranze accettabili. $\mathrm{E}$ a me sembra semmai più verosimile l'eventualità contraria di una congiura «anti-presidenziale» intesa a ritardare al massimo l'inizio della fase presidenziale. Ammettiamo, sempre arguendo, che in tal caso il presidente sia insediato per un anno solo. Nemmeno in questo caso mi strapperei $\mathrm{i}$ capelli.

Ma perché escludere, mi si chiederà, che la congiura anti-presidenziale riesca a impedire del tutto lo scatto? In dannatissima ipotesi non lo escludo. Ma conto su due constituencies, su due coagulazioni di forze e di interessi. Se si andrà a formare una congiura anti-presidenziale, si andrà anche a formare - come Panebianco teme e come io, invece, spero - il partito del presidente. La congiura anti-presidenziale si fonda sugli interessi costituiti e sul danno che subiranno al momento della loro messa in mora; e il partito del presidente si costituirà, prevedo, sugli interessi esclusi e sul rigetto, nell'opinione pubblica, del parlamentarismo dilatorio. Nel mio scenario, dunque, ogni giocatore «lascerà giocare» l'altro perché ha convenienza a minimizzare il rischio di perdere il gioco successivo.

Secondo me il punto delicato della proposta non è nella sua pericolosità e nemmeno nella sua aggirabilità; è su come attivare - anzi riattivare - un «controllo parlamentare» che sia tale senza bloccare il «governo presidenziale». Anche se il governare legiferando è drasticamente da ridurre ${ }^{17}$, vi sono pur sempre atti

17 Sulla deformazione posta in essere dal «governare legiferando» vedi sub «Parla- 
di governo che debbono avere forma di legge e che debbono «passare» come leggi. Come si fa?

La possibilità di governare per decreto deve restare perché altrimenti l'ostruzionismo parlamentare diventa pagante; ma deve restare come ultima ratio. Prima si devono esperire le vie normali, il cui iter si potrebbe configurare (tutto quel che andrò a suggerire è meramente «tentativo») come appresso. Nella fase presidenziale - e come in tutti i presidenzialismi - l'iniziativa compete in esclusiva al presidente e al suo governo. Seguiamo, ciò posto, il cammino di un disegno di legge. Dovrà andare alla commissione parlamentare competente di una camera, o di tutte e due? Io manterrei la doppia approvazione per la fase parlamentare e la sospenderei per la fase presidenziale. Dunque, niente «spoletta». Il disegno di legge andrà, seguendo criteri da prestabilire, a una sola commissione la cui opzione è di approvarlo oppure di rinviarlo al presidente con proposte di emendamento debitamente spiegate e giustificate da un testo di accompagnamento. Ridetto all'inverso, le commissioni parlamentari non possono, in prima istanza, né respingere in toto, né approvare un testo modificato. A questo punto il presidente può negoziare informalmente il tutto, oppure accettare alcuni emendamenti e rifiutarne altri. Dopodiché il disegno di legge diciamo definitivo va questa volta in aula, la cui sola opzione è di approvare o respingere (votando, in tal caso, anche un testo di accompagnamento che motiva il rifiuto). Se respinge, allora al presidente viene consentito di ricorrere al decreto legge (che deve anche lui, a sua volta, motivare); decreto legge che non decade, visto che potrà essere fatto decadere, in sostanza, da una nuova legge del nuovo parlamento (a gestione parlamentare) che verrà. Infine, per evitare ostruzionismi dilatori si dovranno prestabilire limiti di tempo entro i quali commissioni e aula dovranno esaminare e concludere: altrimenti il loro silenzio, o la loro inazione, diventa assenso.

Non sfuggirà che l'innovazione nella quale confido molto è che nulla deve più avvenire soltanto «tra le righe». Nei procedimenti che sono andato ipotizzando tutti gli attori - presidente incluso - devono sempre motivare il proprio dissenso, e le proprie proposte o controproposte, con un testo che le renda trasparenti e intelligibili (un allegato da pubblicare sulla Gazzetta Uffi-

mento» in Sartori (1990, 181-191), dove mi soffermo anche sull'atrofia della funzione di controllo dei parlamenti. 
ciale come parte integrante della legislazione stilata in articoli). Le «leggine con fotografia» che sono tali devono essere difese o affossate come tali. E le infinite interpolazioni surrettizie (tra le righe) che alle volte sfuggono persino agli addetti ai lavori non devono più sfuggire a nessuno. Insomma, il sottobanco deve essere messo in luce sul banco. Per un parlamento è facilissimo votare no; ma è assai meno facile concordare e votare un testo che lo motivi. Se quel testo risulterà debole e pretestuoso, darà man forte al presidente nel procedere per decreto; ma se sarà ben motivato $\mathrm{e}$ convincente, sarà il presidente a dover stare attento.

Basta così? Convengo che non basta. Il rinvio alla legislatura che verrà può risultare un fermo tardivo. $\mathrm{E}$ tutte le remore al mondo non tolgono che il potere di decretazione si debba anche imbattere in un limite. Per esempio, che i decreti presidenziali decadano illico et immediate su voto contrario di una maggioranza qualificata delle camere. Il problema è — come spesso è — di stabilire quale maggioranza sia qualificata. Prestabilirla con percentuali fisse è «senza logica»; talvolta ci regala una percentuale troppo bassa che scatta troppo facilmente; talvolta una percentuale troppo alta che non scatta quando davvero occorre. La mia idea è di renderla variabile determinandola, legislatura per legislatura, così: la maggioranza qualificata è data dal totale di ogni camera, meno i rappresentanti dello stesso partito che ha eletto il presidente. A questo modo un presidente il cui partito ottiene solo il $10 \%$ viene rinforzato: per bloccarlo occorre il $90 \%$ dei voti parlamentari. Viceversa un presidente espresso da un partito forte che vince, mettiamo, il $40 \%$ può essere bloccato dal $60 \%$. La «logica» di questo criterio è duplice. Primo, la forza costituzionale del presidente resta costante, e cioè non varia in funzione delle distribuzioni contingenti delle rappresentanze parlamentari. Secondo, l'ago della bilancia risiede nel partito che elegge il presidente, e così per la ragione (spero buona) che un presidente che fa bene premia anche il suo partito, che ha pertanto interesse a sostenerlo, mentre un presidente che fa male verrà abbandonato anche dai suoi.

Mi fermo qui perché l'intento è solo di far vedere che una coesistenza costruttiva - tale perché non consente alternative ostruzionistiche - tra controllo parlamentare e decisionismo presidenziale non è un problema di quadratura del cerchio. 
Riferimenti bibliografici

Linz, J. (1991), Democrazia: presidenziale o parlamentare. Fa differenza?, in «Informazioni Arel», maggio.

Sartori, G. (1982), Teoria dei partiti e caso italiano, Milano, SugarCo.

- (1990), Elementi di teoria politica, Bologna, II Mulino, $2^{\mathrm{a}}$ ediz.

- (1991), Né presidenzialismo né parlamentarismo, in «Informazioni Arel», maggio.

Valenzuela, A. (1991), Politica di partito e fallimento del presidenzialismo in Cile, in «Informazioni Arel», maggio. 